\title{
Severe head dysgenesis resulting from imbalance between anterior and posterior ontogenetic programs
}

Emmanuelle Grall', Victor Gourain (10) ${ }^{2}$, Asmaa Nairr ${ }^{1}$, Elisabeth Martin ${ }^{1}$, Marie-Christine Birling ${ }^{3}$, Jean-Noël Freund (1) ${ }^{1}$ and Isabelle Duluc (iD)

\begin{abstract}
Head dysgenesis is a major cause of fetal demise and craniofacial malformation. Although mutations in genes of the head ontogenetic program have been reported, many cases remain unexplained. Head dysgenesis has also been related to trisomy or amplification of the chromosomal region overlapping the CDX2 homeobox gene, a master element of the trunk ontogenetic program. Hence, we investigated the repercussion on head morphogenesis of the imbalance between the head and trunk ontogenetic programs, by means of ectopic rostral expression of CDX2 at gastrulation. This caused severe malformations affecting the forebrain and optic structures, and also the frontonasal process associated with defects in neural crest cells colonization. These malformations are the result of the downregulation of genes of the head program together with the abnormal induction of trunk program genes. Together, these data indicate that the imbalance between the anterior and posterior ontogenetic programs in embryos is a new possible cause of head dysgenesis during human development, linked to defects in setting up anterior neuroectodermal structures.
\end{abstract}

\section{Introduction}

Head dysgenesis during embryonic development is a major cause of fetal demise occurring in $\sim 1 / 250$ conceptuses ${ }^{1}$. It covers a large spectrum of craniofacial and brain malformations and involves mutations in genes of the anterior ontogenetic program as well as environmental conditions. Yet, a great number of cases still remain unexplained ${ }^{2}$. During embryogenesis, the head is the first body part to form around gastrulation from resident anterior epiblast cells ${ }^{3,4}$. Unlike the head, the trunk is newly built by progressive posterior addition of

\footnotetext{
Correspondence: Jean-Noël. Freund (jean-noel.freund@inserm.fr) or Isabelle Duluc (isabelle.duluc@inserm.fr)

${ }^{1}$ Université de Strasbourg, Inserm, IRFAC/UMR-S1113, FMTS, 67200 Strasbourg, France

${ }^{2}$ Karlsruhe Institute of Technology, Institute of Toxicology and Genetics, 76021 Karlsruhe, Germany

Full list of author information is available at the end of the article.

These authors contributed equally: Jean-Noël Freund, Isabelle Duluc

Edited by A. Stephanou
}

tissue from the posterior epiblast through the primitive streak to get the three germ layers ${ }^{5}$. The posterior ontogenetic program notably differs from the head program by the involvement of an intricate network of homeobox transcription factor genes including the caudal-related paraHox genes $C d x 1 / 2 / 4$, the POU-homeobox gene Oct4 and the genes of the four Hox clusters ${ }^{6,7}$. Cdx $1 / 2 / 4$, whose nested expression is limited rostrally in rhombomere 3 , are crucial for maintaining the pool of posterior elongation progenitors, so that $C d x$-null murine embryos fail developing body structures beyond the occiput ${ }^{8}$. Oct4 sustains progenitor pluripotency ${ }^{9}$, and $H o x$ genes ${ }^{10}$, together with $C d x 1 / 2^{11,12}$, provide the growing posterior tissues their anteroposterior (AP) positional information. Hox clusters genes, limited anteriorly in rhombomere $2^{13}$, exhibit spatial and temporal colinearity ${ }^{6,7}$ under complex regulatory mechanisms involving notably the CDX factors $^{14,15}$.

\section{(c) The Author(s) 2019}

(c) (i) Open Access This article is licensed under a Creative Commons Attribution 4.0 International License, which permits use, sharing, adaptation, distribution and reproduction cc) in any medium or format, as long as you give appropriate credit to the original author(s) and the source, provide a link to the Creative Commons license, and indicate if changes were made. The images or other third party material in this article are included in the article's Creative Commons license, unless indicated otherwise in a credit line to the material. If material is not included in the article's Creative Commons license and your intended use is not permitted by statutory regulation or exceeds the permitted use, you will need to obtain permission directly from the copyright holder. To view a copy of this license, visit http://creativecommons.org/licenses/by/4.0/. 
Among the three $C D X$ paralogues, $C D X 2$, located on human chromosome 13, is important not only for posterior elongation and patterning at gastrulation but also earlier in the trophectoderm ${ }^{16,17}$ and later in the gut ${ }^{18,19}$. Noteworthy, although $C D X 2$ is not normally expressed in head progenitors, head dysgenesis has been frequently associated with the trisomy of chromosome 13 (Patau syndrome $)^{20}$ or with partial trisomy of the long arm of this chromosome including the region q12.2 that overlaps the $C D X 2$ locus $^{21,22}$. The Patau syndrome is a rare and dramatic disease whose prevalence is estimated at 1:12,000 to $1: 29,000$ in newborns with a median survival time of 6-10 days ${ }^{23}$.

On this basis, the link between the amplification of the locus containing the posterior ontogenetic gene, $C D X 2$, and the occurrence of rostral malformations led us to investigate the repercussion on head morphogenesis of disturbing the balance between anterior and posterior developmental programs, by means of ectopically expressing $C D X 2$ rostrally at gastrulation.

\section{Results \\ Head dysgenesis caused by rostral ectopic expression of CDX2}

Mice designed for inducible expression of the human $C D X 2$ homeobox gene, the RsCDX2 mice, were generated by inserting into the Ros 26 locus the human $C D X 2$ cDNA preceded by a loxP-flanked transcriptional stop cassette (Fig. 1A). Ectopic expression of the CDX2 protein rostrally to its anterior limit in rhombomere 3 was achieved using these mice crossed with Sox $2 \mathrm{Cr} e^{E R T 2}$ mice expressing $\mathrm{Cre}^{\mathrm{ERT} 2}$ in the whole epiblast at gastrulation, while the pregnant females received a single injection of Tamoxifen at day 6.5 post-coitum. The procedure was validated by demonstrating the rostral expression of Tomato protein in RosaCAG ${ }^{\text {tdTomato }:: S o x 2 C r e^{E R T 2}}$ embryos (Fig. 1Ba), mainly at the level of the neuroepithelium, neural crest derived cells and ectoderm, but not in the cephalic mesenchyme (Fig. 1Bb). It was then successfully applied to RsCDX2::Sox2Cre $e^{E R T 2}$ embryos to trigger ectopic expression of CDX2 in these tissues, as shown by whole-mount immunohistochemistry and immunostaining on tissue sections (Fig. 1C) and by RTqPCR (Fig. 1D). Although no macroscopic phenotype was displayed in mutants before E9.5, head dysmorphology characterized by a flattened anterior aspect appeared around E10.5, worsened at E12.5, and led to profound deformities at E15.5 (Fig. 1E; Supplementary Figure S1): the frontonasal process was missing leading to exencephaly, eyes were absent or limited to rudimentary structures and the maxillary branch of the first pharyngeal arch failed to merge axially. Half of the mutants also exhibited preaxial forelimb polydactyly (Supplementary Figure S1).

\section{Rostral ectopic expression of CDX2 perturbs the anterior ontogenetic program and induces elements of the posterior program}

Transcriptome analysis of the head of E10.5 control and mutant RsCDX2::Sox2Cre ${ }^{E R T 2}$ littermates identified 532 differentially-expressed genes (Supplementary Table S1) falling into 3 categories by Principal Component Analysis (Fig. 2A, B; Supplementary Table S2 sheet 1). Category 1 corresponded to the 143 genes downregulated in mutants and functional annotation clustering revealed that it organized into 3 clusters respectively associated with the Gene Ontology (GO) terms Axon/Dendrite; Cerebral cortex neuron differentiation/Negative regulation of neuron differentiation; and Sequence-specific DNA binding. Several of the downregulated genes encoding DNA binding factors are crucial for head development like Otx1 (brain and sensory organ development), Foxg1, Nkx2.1 and Six3 (telencephalon formation), Tal2 (midbrain formation), Six2 (growth of the cranial base), Ascl1 and $M y b$ (neuronal commitment and progenitor proliferation), Nr2e1 (forebrain and retina stem cell determination), Six3, Six6, Eya4, Vax1 and Vsx2 (eye morphogenesis), Tcf7L2 (radial migration of cortical neurons), Fezf2 and Bcl11a (subcerebral neurons specification), Helt (GABAergic neurogenesis), Rora (Purkinje cells development), and Zbtb2O (hippocampal neuronal determination). Thus, the head ontogenetic program is largely perturbed following the rostral induction of $C D X 2$ expression. Unlike the category 1 , the categories 2 and 3 covered the 389 upregulated genes. Category 2 (364 genes) subdivides into 5 clusters associated with the GO terms Extracellular matrix region; Anterior-posterior patterning/Sequence-specific DNA binding/Regulation of transcription; Collagen; and Wnt signaling. Category 3 (25 genes) presents the cluster with the highest enrichment score, related to the GO terms Anterior-posterior patterning/Sequence-specific DNA binding/Regulation of transcription/Embryonic skeletal morphogenesis. To go further into the characterization of the 389 genes upregulated by rostral ectopic expression of $C D X 2$, the list of genes of the categories 2 and 3 was compared with two other experimental conditions where the $C d x$ function is altered $^{14}$ : the genes downregulated in E8 $C d x$-null embryos in which the trunk program is arrested, and the genes upregulated in epiblast stem cells cultured with a Wnt agonist plus Fgf8 to recapitulate the onset of the posterior program including $C d x 2$ (Fig. 2C, D; Supplementary Table $\mathrm{S} 2$ sheet 2 ). Several genes arose by pairwise comparison while the 3 lists exhibited a set of 11 genes in common. Remarkably, the genes identified in the pairwise comparisons as well as the 11 genes in common in the 3 experimental conditions are related to the GO terms Anterior-posterior patterning/DNA binding/Regulation of transcription. This highlights that rostral CDX2 


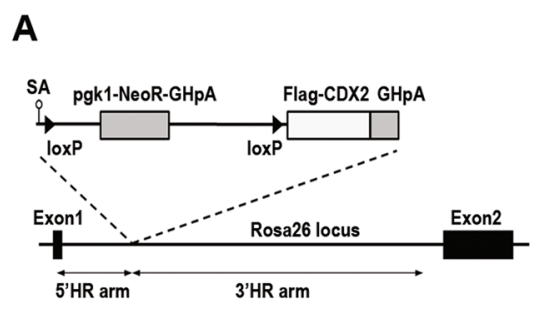

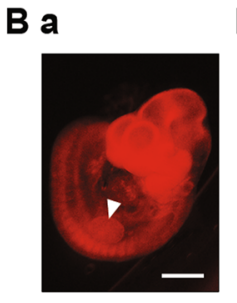

Tomato

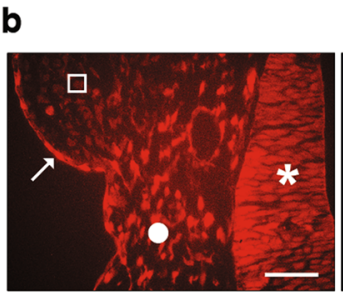

Tomato

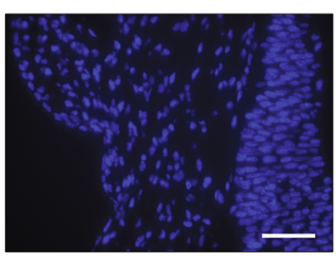

Dapi
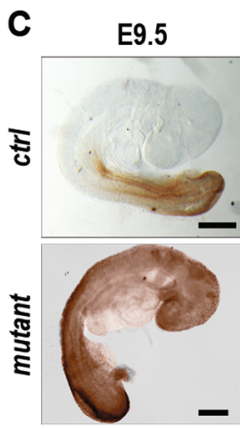

E10.5

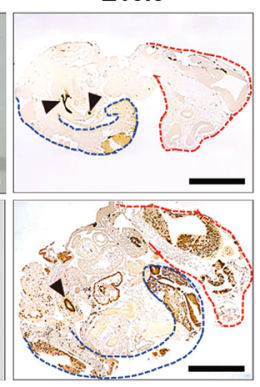

D

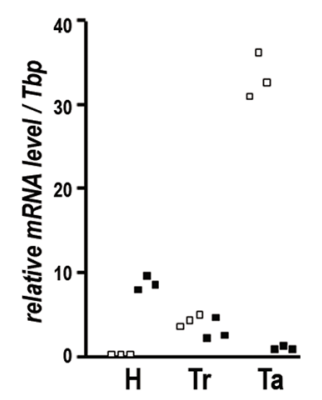

E

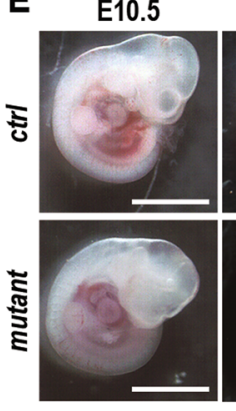

E12.5

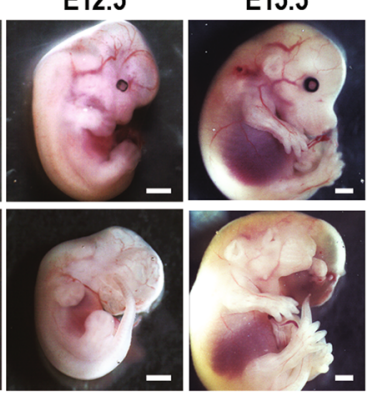

Fig. 1 Morphologic alterations caused by rostral ectopic expression of CDX2. A The RsCDX2 allele; SA: Splicing Acceptor site; GHpA: polyadenylation site of the human Growth Hormone gene. Ba Tomato fluorescence emitted by E10.5 RosaCA G $^{\text {tdTomato }}:$ :Sox2Cre $e^{\text {ERT2 }}$ embryos exposed to Tamoxifen at E6.5. The arrowhead points to the anterior limb bud. Bar: $500 \mu \mathrm{m}$. b Transversal sections in the telencephalon showing Tomato fluorescence emission at the level of the neuroepithelium (asterisk), neural crest derived cells (closed circle) and ectoderm (arrow) but not in the cephalic mesenchyme (open square). Bars: $50 \mu \mathrm{m}$. C Immunodetection of the CDX2 protein in whole-mount preparations of E9.5 control (ctrl) and RsCDX2::SOX2Cre ${ }^{E R T 2}$ (mutant) littermates, and in sections of E10.5 control and mutant embryos. Red and blue dotted lines mark head and tail, respectively. Arrowheads show the endogenous Cdx2 in the gut endoderm. Bars: $500 \mu \mathrm{m}$. D Relative RNA levels by RTqPCR of the transcripts for endogenous Cdx2 (open squares) and for the CDX2 transgene (black squares) in the head (H), trunk ( $\mathrm{rr}$ ) and tail (Ta) of 3 mutant embryos at E10.5. E Morphology of E10.5, E12.5 and E15.5 control and mutant embryos. Bars: $1 \mathrm{~mm}$

induction not only perturbs the normal anterior ontogenetic program of the head, but it also turns on elements of the posterior program normally active in the developing trunk.

\section{Ectopic CDX2 turns on the expression of all central Hox clusters genes}

Transcription factor genes represent 19.7\% (105/532) of the genes deregulated by ectopic expression of CDX2 (Supplementary Table S2 sheet 3). Their relevance is supported by the significant enrichment in DNA binding sites for 45 of them $(42.9 \%$; FDR $<0.02)$ in the $-2000 /+$ 50 -bp promoters of the whole set of deregulated genes (Fig. 3A). These 45 factors fall into 9 groups according to their DNA binding domain. Homeobox genes, known to be crucial in morphogenesis, represent 48 of the 105 deregulated transcription factor genes and correspond to 5 of the 9 groups identified by DNA binding sites enrichment. Importantly, these homeobox genes include the majority (24/39) of the genes of the four Hox clusters, especially those involved in trunk patterning (Fig. 3B). Indeed, while transcripts of the anterior genes Hoxa1, $-a 2,-b 1$, and $-b 2$ were already present in the head of control embryos, consistent with their normal anterior limit in the hindbrain, all the 17 central cluster genes from position 3 to 9-10, also belonging to the set of 25 genes of the category 3 in the Principal Component Analysis, were unexpressed in the head of control embryos but turned on in the mutants. More posterior Hox genes at positions $11-13$, controlled by $G d f 11$ instead of $C d x$ in the trunktail transition ${ }^{24}$, remained silent. Chromatin immunoprecipitation (ChIP) targeting the Cdx2-binding site previously identified and functionally characterized upstream of the Hoxa5 gene ${ }^{15,25}$ showed occupancy by the endogenous $\mathrm{Cdx} 2$ protein in the trunk but not in the head of control E10.5 embryos, as expected; however, the CDX2 protein was actually bound to this site in the head of RsCDX2::Sox2Cre ${ }^{E R T 2}$ littermates (Fig. 3C), demonstrating the direct interaction of $\mathrm{CDX} 2$ with the Hoxa5 promoter when this gene becomes turned on in the head of mutant embryos. Thus, ectopic expression of $C D X 2$ is able to switch on central Hox genes in the head, similar to what happens during trunk morphogenesis.

\section{Ectopic CDX2 perturbs cell fate and cell interactions}

Histology was analyzed in mutants between E9.5 and E15.5 and representative pictures of embryos at day 10.5 and 13.5 are illustrated in Fig. 4A, B. As shown at E13.5, 
A

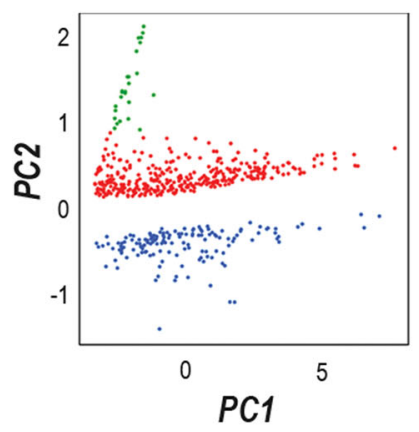

C

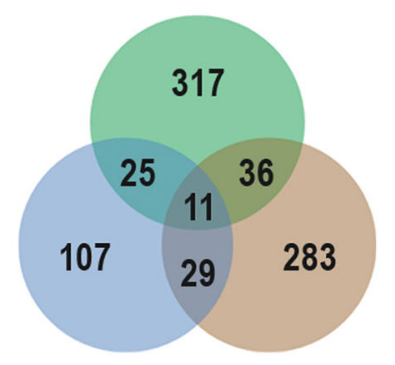

B
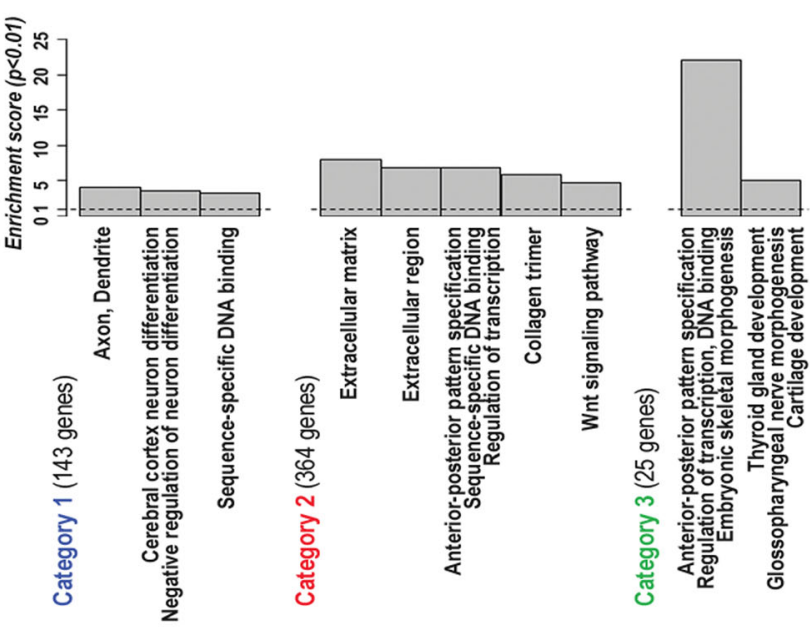

D

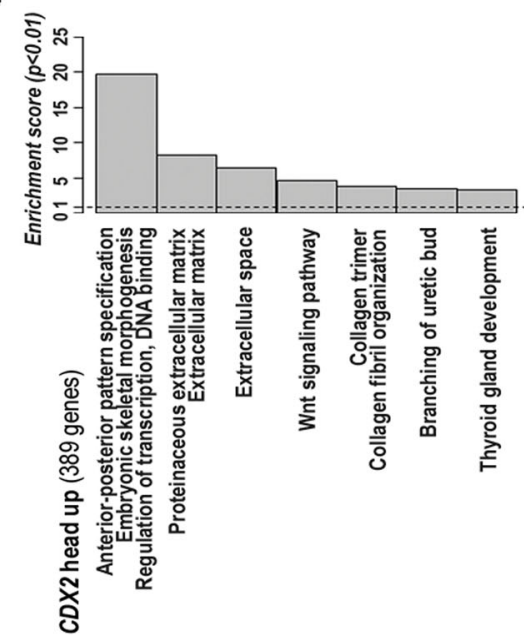

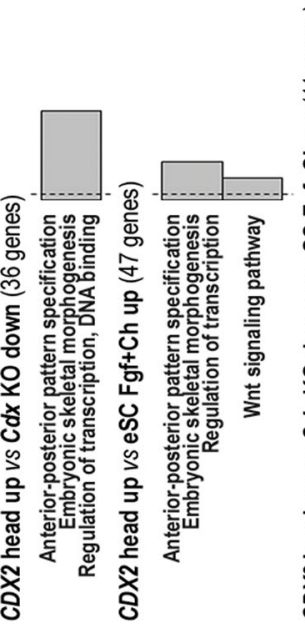

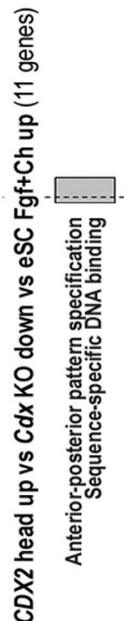

Fig. 2 Gene modulation caused by rostral ectopic expression of CDX2. A Principal Component Analysis of the 532 differentially expressed genes by rostral ectopic expression of CDX2 in the head of E10.5 embryos (absolute Log2FC $>0.4$, adjp < 0.05). B Functional Annotation Clustering (Enrichment score $>3 ; p<0.01$ ) of the 3 gene categories defined by Principal Component Analysis. pvalues were corrected with FDR multiple testing method. C Venn representation of the genes upregulated by ectopic expression of CDX2 in the head (green), of those upregulated in embryonic stem cells treated with CHIR99021 plus Fgf8 (brown), and of those downregulated in Cdx-null embryos (blue). D GO term representation (Enrichment score $>3 ; p<0.01)$ for the lists of common genes displayed by the comparative analysis in $\mathbf{D}$

profound tissue disorganization occurred rostrally where ectopic CDX2 was the highest in the neuroepithelium. The shape of the diencephalon and third ventricle was altered together with the atrophied structures of the telencephalon and lateral ventricles; the choroid invagination was missing and eyes were absent or poorly formed. Craniofacial structures were also missing. These alterations connected with the reduced level or absence of forebrain transcription factors, as shown by immunohistochemistry at E10.5 for Nkx2.1 and Foxg1 in the telencephalon (Fig. 4) and even more pronounced at E13.5 for Foxg1 in the lateral ventricles, Nkx2.1 in the medial ganglionic eminence, Otx2 in the medial ganglionic eminence and choroid plexus and Six3 in the diencephalon (Fig. 4D). The decrease of these proteins was in line with the RNAseq data. Reciprocally, RNAseq had revealed the increase of mRNAs for transcription factors normally expressed in the trunk like Hox genes, and/or caudally to the forebrain in the mid/hindbrain, such as Gbx1, Gbx2, En1, Foxb1, Foxd3 and Hes3. Actually, the Hox 5 protein was detected in the atrophied lateral ventricles of E13.5 mutants, namely more rostrally than the normal anterior limit of Hox genes (Fig. 4E), and Gbx1 also was shifted rostrally in the telencephalon of E10.5 mutants compared to its normal expression caudal to hindbrain rhombomere 2 in control embryos (Fig. 4F).

Cell interactions also contribute to the morphological defects caused by ectopic CDX2, as exemplified in eye remnants (Fig. 5A). In a few E12.5 mutants, rudimentary ocular structures developed when the tip of the optic stalk 


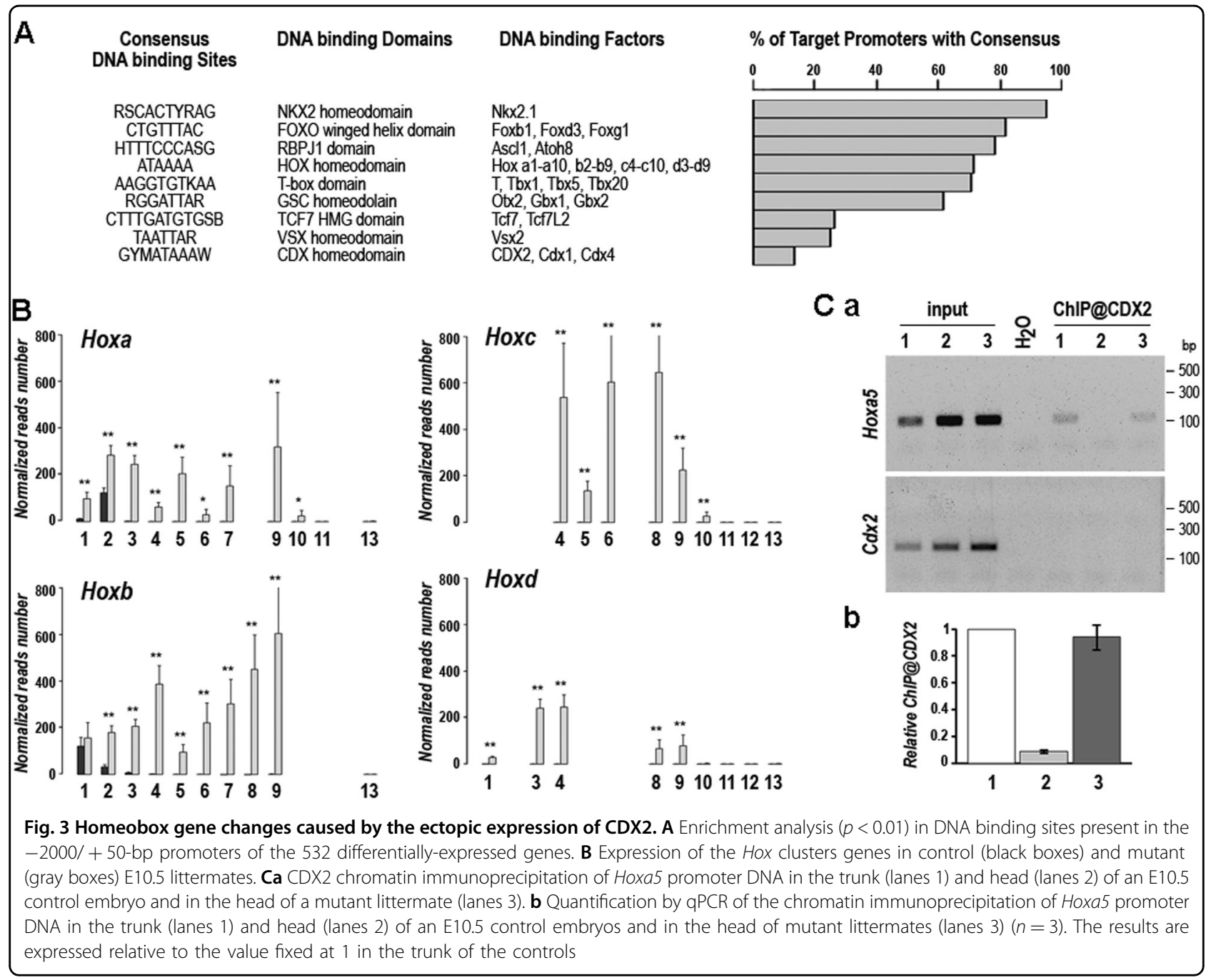

remained CDX2-free. The neuroepithelium differentiated into a layer of Otx2-positive retinal pigment cells but failed to invaginate into optical cuff and to form the Sox2positive neural layer. Concomitantly, the surface ectodermal layer facing the Otx2-positive neuroepithelium, also ectopically expressing CDX2, did not invaginate into the lens while maintaining p63 in contrast to its normal loss in the presumptive lens ectoderm ${ }^{26}$. Thus, ectopic CDX2 not only perturbs neuroepithelium development, but it also alters neuroepithelial-ectodermal interactions needed for eye morphogenesis.

Together with forebrain alterations, RsCDX2::Sox2$C r e^{E R T 2}$ mutants exhibited strong defects in the frontonasal process. The rostral shift in Hox expression corroborated the incompatibility of these genes with craniofacial development ${ }^{27-29}$. While the frontonasal process develops from the colonization of streams of diencephalic and anterior mesencephalic neural crest cells around E8.5-E9.5 ${ }^{3}$, RNAseq data revealed mRNA changes for members of genes families involved in cell guidance during neural crest cell migration including Erbb3/4, Sema3b/6b, Nrp1/2 and Epha2/a3/a5/b1. Sox10 immunostaining used to label cells of neural crest origin showed a reduction of cell number in E10.5 mutants compared to controls, accompanied by cleaved-Caspase- 3 staining and a low rate of phospho-Histone-3-positive cells (Fig. 5B). Thus, ectopic CDX2 compromises the migration and survival of neural crest cells intended to form the face. In addition, in absence of frontonasal process, the rostralmost wall of the forebrain was profoundly remodeled, as illustrated at E13.5 (Fig. 6A). The resulting structure was made of a compact, yet vascularized, cell mass covered by a single-layered CDX2-free but p63- and Sox9-positive epithelium. The cell mass strongly expressed CDX2, was proliferative, but devoid of all neuroepithelial factors tested here (Foxg1, Gbx1, Nkx2.1, Otx2, Six3, Sox2, Sox9) and also Sox10, suggesting no neural crest cell origin. Earlier stages at E11.5-E12.5 revealed the dynamics of the 


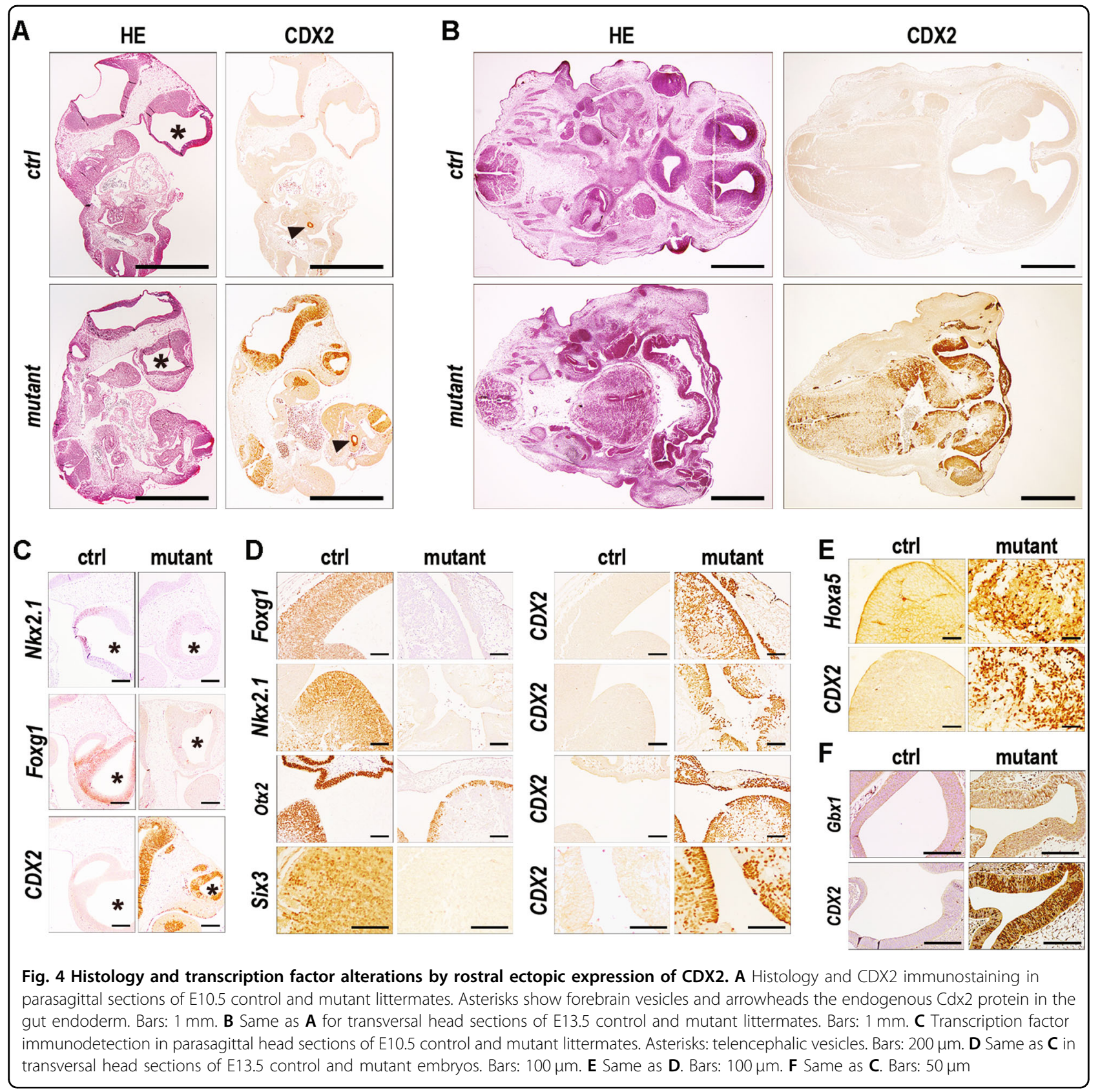

process where the Otx2-positive neuroepithelium expressing CDX2 became patchy and progressively replaced by CDX2-positive and Otx2-negative cells (Fig. $6 \mathrm{~B})$. This replacement was associated with the release of cellular material from the neuroepithelium into the telencephalic ventricles (Fig. 6C), without evidence of cleaved-Caspase- 3 apoptosis. Thus, ectopic expression of CDX2 compromises the development of the facial neural crest-derived mesenchyme, associated with the disintegration of the neuroepithelium and its replacement by cells having lost neuroepithelial properties.

\section{Discussion}

Although the impact of the loss of $C D X$ function during embryonic development has been well documented, deciphering the primary role of this gene family in trunk elongation and patterning ${ }^{8,30}$, the effect of the abnormal anterior gain of function has not been studied so far in vertebrates. In $D$. melanogaster, forced expression of the caudal orthologue in the anterior pole at the syncytial embryonic stage induces head malformations, yet without homeotic changes ${ }^{31}$. Here, we developed the model of ectopic expression of $C D X 2$ in rostral tissues of mouse 


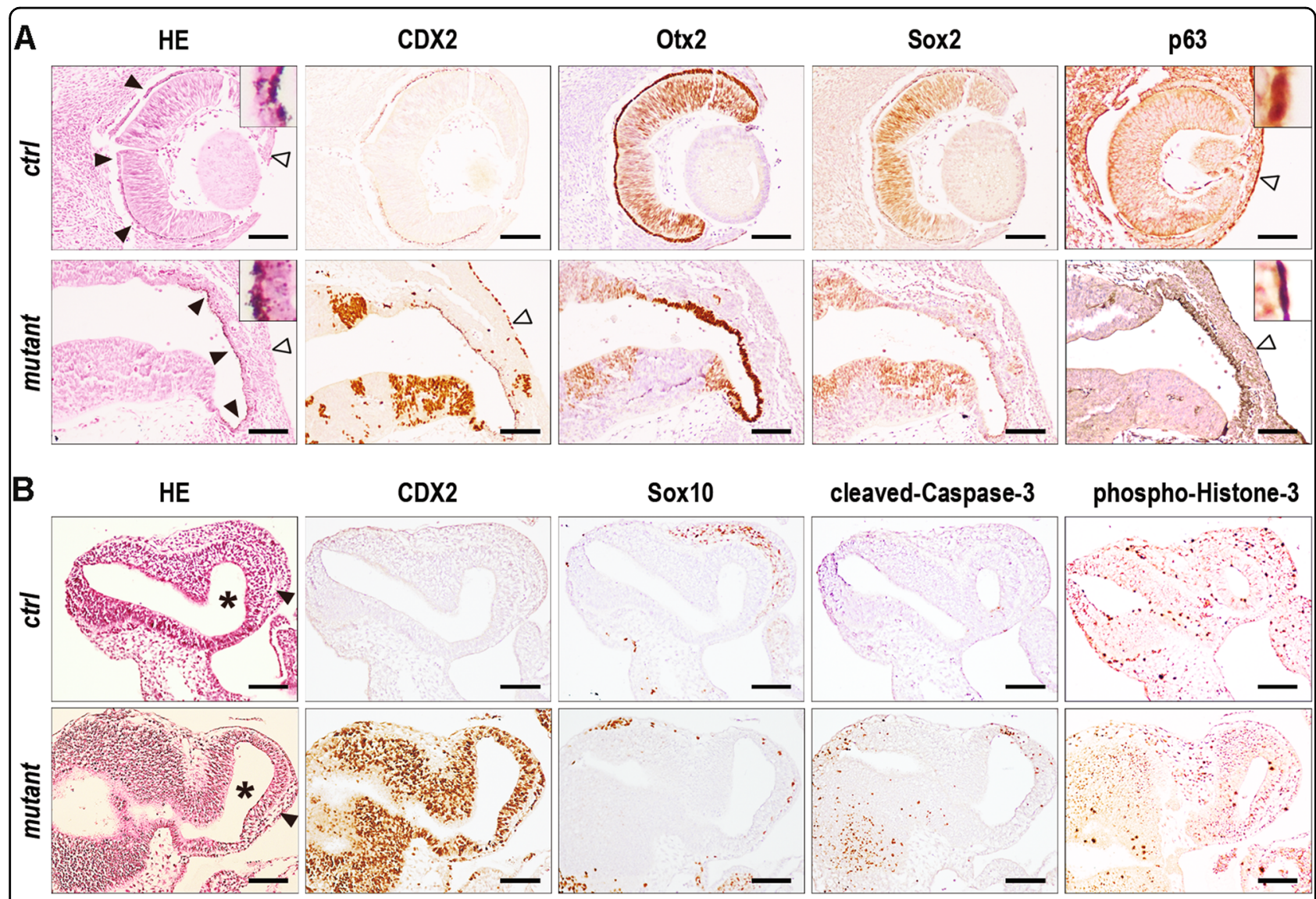

Fig. 5 Eye and frontonasal malformations caused by rostral ectopic expression of CDX2. A Serial transversal sections of eyes and ocular remnants of E12.5 control and mutant littermates. Inserts are higher magnification of the retinal pigment epithelium and of the p63-positive ectodermal epithelium. Black and open arrowheads show retinal pigment cell layer and ectodermal layer, respectively. Bars: $100 \mu \mathrm{m}$. B Serial parasagittal sections of the frontonasal process (arrowheads) of E10.5 control and mutant littermates. Asterisks: telencephalic ventricles. Bars: $200 \mu \mathrm{m}$

embryos to investigate the consequences of disturbing the balance between the anterior and posterior ontogenetic programs. The resulting embryos present with huge brain and craniofacial dysgenesis recapitulating several aspects of human malformations including holoprosencephaly. These malformations are associated with the decrease of important genes of the anterior program together with the rostral onset of elements of the posterior program among which are the trunk Hox genes. Unlike the endoderm, where ectopic CDX2 induces a homeotic shift from foregut to midgut-like properties ${ }^{32}$, in the rostral neuroepithelium this factor leads to missing head structures associated with perturbations in cell movement, proliferation and death, in particular at the level of neural crest cells and for the formation of eyes. This likely results from the interference between the endogenous anterior program of the head and the ectopic posterior program driven by the CDX2 protein known from gut studies to be a regulator of active chromatin organization ${ }^{33,34}$.

This work is highly relevant as regards head dysgenesis diseases in humans because a number of cases, among which the Patau syndrome, are associated with copy number amplification of the chromosome 13 locus overlapping $C D X 2$. Thus, this study highlights that, in addition to single gene mutations in the head ontogenetic program $^{2}$, the imbalance between head and trunk programs around gastrulation could be a novel basis of head dysgenesis. The mouse phenotype resulting from the forced expression of $C D X 2$ anteriorly above the normal anteriorposterior gradient, recapitulates observations made in human, including not only brain malformations, eye defects and frontonasal dysmorphology but also forelimb polydactyly. Together, these data open the way to further fetopathological studies to address in humans the importance of the imbalance between the anterior and posterior ontogenetic programs in head dysgenesis diseases of still unknown origin.

\section{Material and methods Mice and treatments}

All the mouse experiments were performed in the certified animal facility (number $\mathrm{H}-67-482-21$ ) according to 


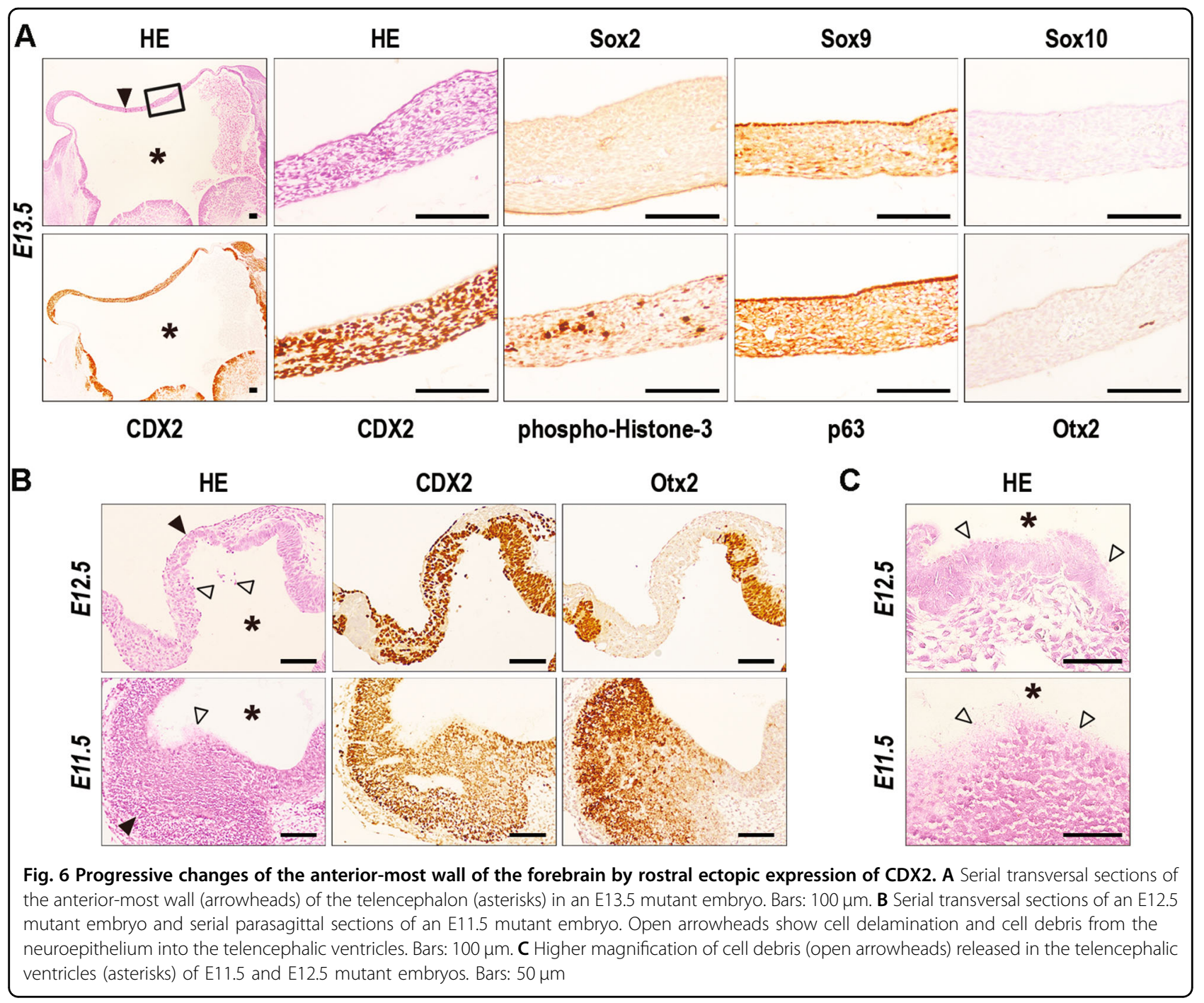

the protocol approved by the French Ministry of Agriculture under the permit APAFiS\#833.

The RsCDX2 mouse was generated by inserting into the Rosa26 locus the cassette made of a Splicing Acceptor site, an excisable fragment flanked by loxP sites and the FLAG-tagged form of the human CDX2 $\mathrm{cDNA}^{35}$. The excisable fragment contains the transcription unit formed by the $p g k 1$ promoter, the neomycin-resistance coding sequence and the Growth Hormone polyadenylation site. The RsCDX2 mouse was generated at the Mouse Clinic Institute (Illkirch, France). A schematic representation of the RsCDX2 allele is shown in Fig. 1Aa.

Sox $2 \mathrm{Cre}^{E R T 2}$ (stock number 017593) ${ }^{36}$ and Rosa$C A G^{\text {tdTomato }}$ (stock number 007909) ${ }^{37}$ mice were from the Jackson laboratory. Tail DNA and extraembryonic membrane DNA were respectively used for genotyping adult mice and embryos by PCR with the following primers: RsCDX2 allele: GTGGTTTGTCCAAACTCATCA/
CACGTGGTAACCGCCGTAGTC; Sox2Cre $e^{E R T 2}$ allele: GCGGTCTGGCAGTAAAAACTATC/GTGAAACAG CATTGCTGTCACTT; RosaCAG ${ }^{\text {tdTomato }}$ allele: GGCAT TAAAGCAGCGTATCC/CTGTTCCTGTACGGCAT GG.

Pregnant females of RsCDX2::Sox $2 \mathrm{Cr} e^{E R T 2}$ or Rosa$C A G^{\text {tdTomato }}::$ Sox $2 C r e^{E R T 2}$ intercrosses were given a single intraperitoneal injection of $2 \mathrm{mg}$ of Tamoxifen in corn oil at day 6 after vaginal plug detection (E6.5 post coitum).

In all the figures illustrated for this work, control (ctrl) and mutant ( $R S C D X 2:: S o x 2 C r e^{E R T 2}$ ) embryos were littermates; the controls were either wild-type or RsCDX2 or Sox 2 Cre $e^{E R T 2}$ embryos, giving identical results. Littermates with no detectable head expression of ectopic CDX2 protein by immunohistochemistry were excluded. For each developmental stage, embryos from 3 littermates with ectopic CDX2 expression were analyzed. No randomization was used. Phenotypic analyses were performed in blind. 


\section{Histology and immunohistochemistry}

Whole-mount immunohistochemistry was performed on E9.5 embryos as described ${ }^{38}$. For this purpose, embryos were fixed overnight in DMSO/methanol (1:4), rehydrated in PBS, blocked in $2 \%$ dry milk, $0.5 \%$ Triton X100 and incubated overnight with rabbit anti-CDX2 antibody CDX2 (Abcam; ab76541; RRID:AB_1523334, dilution 1:5000). HRP-labeled goat anti-rabbit antibody (Vector Laboratories; PI-1000; RRID: AB_2336198, dilution 1:500) was added overnight and revealed with DAB. Embryos were cleared with Methyl Salicylate before observation.

For immunohistochemistry on tissue sections, E10.5 to E15.5 embryos were fixed with 4\% PFA, embedded in paraffin and sectioned $(5 \mu \mathrm{m})$ before staining with hematoxylin-eosin (HE) or by immunohistochemistry, as described $^{39}$. Antibodies were: rabbit anti-cleavedCaspase-3 (R\&D System; AF835; RRID:AB_2243952, dilution 1:500), rabbit anti-CDX2 (Abcam; ab76541; RRID:AB_1523334, dilution 1:10,000), rabbit anti-Foxg1 (Abcam; ab18259; RRID:AB_732415, dilution 1:1000), rabbit anti-Gbx1 (dilution 1:5000) ${ }^{40}$, mouse anti-Hoxa5 (Santa Cruz; sc-515309, dilution 1:250), mouse anti-Nkx2.1 (Thermo Fischer; MA5-13961; RRID: AB_10984070, dilution 1:500), rabbit anti-Otx2 (Abcam; ab183951, dilution 1:250), rabbit anti-p63 (Abcam; ab124762; RRID:AB_10971840, dilution 1:2000), rabbit anti-phospho-Histone-3 (Millipore; 06-570; RRID: AB_310177, dilution 1:1000), guinea pig anti-Six3 (Rockland; 200-201-A26S; RRID:AB_1961548, dilution 1:1000), mouse anti-Sox2 (R\&D System; MAB2018; RRID: AB_358009, dilution 1:500), rabbit anti-Sox9 (dilution 1:500) ${ }^{41}$, rabbit anti-Sox10 (Sigma-Aldrich; Ab383R, dilution 1:350). Secondary biotinylated antibodies were from Vector Laboratories and used at the dilution 1:200: goat anti-guinea pig (BA-7000; RRID:AB_2336132), goat anti-rabbit (BA-1000; RRID: AB_2313606), rabbit antigoat (BA-5000; AB_2336126), goat anti-mouse (BA-9200; RRID:AB_2336171).

\section{RNA extraction, RTqPCR and RNAseq analyses}

RNA was individually prepared using TRI Reagent (Euromedex, France) from each of 3 control and 3 mutant E10.5 littermate embryos sectioned to separate the head (sectioned under the binocular loop between the maxillary and mandibular components of the pharyngeal arch 1), the trunk and the tail. Total RNA was treated with DNAse (RQ1 RNAse-free DNAse, Promega Inc.) and its quality was assessed with a Bioanalyzer (Agilent).

For RTqPCR, reverse transcription was performed with $2 \mu \mathrm{g}$ RNA using SuperScript II Reverse Transcriptase (Invitrogen) and Oligo(dT)12-18 primer (Invitrogen). Quantification of the endogenous $C d x 2$ transcript and of the transcript of the CDX2 transgene was performed using the TaqMan Master Mix and gene-specific TaqMan primers sets (TaqMan Gene Expression assays, Life Technologies Applied Biosystems): mouse $C d x 2$, Mm01212280_m1; human CDX2, Hs00230919_m1, and mouse Tbp, Mm00446973_m1. Analysis of the results obtained in triplicate was performed with the 7500 software v2.0.1 (Life Technologies Applied Biosystems) using the relative $\Delta \Delta$ Ct quantification method.

For RNAseq, $1 \mu \mathrm{g}$ of total RNA of each preparation made from the head of 3 control and 3 mutant embryos was used to prepare the sequencing libraries using the Illumina TruSeq stranded mRNA kit, following the supplier's recommendations. The quality and the quantity of the libraries were both assessed on a High Sensitivity chip with a Bioanalyzer. The libraries were diluted to $10 \mathrm{pM}$ to generate clusters in both lanes of a Rapid flowcell (Illumina). Sequencing was performed with a HiSeq1500 (Illumina) in paired-end for a read length of 50b. Cluster mapping was done with the software RTA (v1.13 Illumina). The base calling and the demultiplexing were performed with the software bcl2fastq (v2.17.1.14 Illumina).

The quality of the sequencing reads was assessed with FASTX-toolkit (v0.0.13) and no pre-processing was needed. The alignment of the reads was done with STAR v2. $5^{42}$ against the reference genome of Mus musculus (GRCm38.90). To assess its expression, the human transgene hCDX2 (ENSG00000165556) was added to the reference murine genome as an additional gene and processed as the canonical ones. The aligner STAR was used with default parameters and the alignment was refined with a second pass. The read counting was done with HTSeq v0.6.1 $1^{43}$ in union mode and at gene level. The normalization of gene expression as well as the consistency among biological replicates were both assessed with the R package DESeq2 $1.10 .1^{44}$. Differential expression analysis was also done with DESeq2. Prior to the Wald test, the values of $\log 2$ (Fold Change) were not shrunk. The significantly differentially-expressed genes were identified based on the p-value, corrected with the False Discovery Rate (FDR) multiple testing method, and the $\log 2$ (Fold Change). Genes with an absolute $\log 2$ (Fold Change) $\geq 0.4$ and an adjusted $p$-value $<0.05$ were considered as differentially expressed. All the downstream analyses were done with $\mathrm{R}$ and with $\mathrm{DAVID}^{45}$; Gene Ontology enrichment was done with the one-tailed exact Fisher test and the pvalues were corrected with FDR multiple testing method.

The transcription factor binding motifs in the promoters (from 2000-bp upstream to 50-bp downstream of the transcription starting site) of the differentially expressed genes were analyzed with the software HOMER $\mathrm{v} 4.10^{46}$. Only the known and curated motifs were used for the enrichment analysis. The enriched motifs were 
manually curated comparing with the most up-to-date version of $\mathrm{JASPAR}^{47}$ and Tomtom from the MEME software suite ${ }^{48}$.

\section{Chromatin immunoprecipitation}

E10.5 embryos were harvested and sectioned under the binocular loop between the maxillary and mandibular components of the pharyngeal arch 1 , and above the posterior limbs, to collect and freeze separately in liquid nitrogen the head and the trunk segments, respectively. Extraembryonic membranes were used for genotyping the embryos.

Chromatin immunoprecipitation (ChIP) assay was performed on the head and trunk segments of single control and mutant littermate embryos, using the ChIP IT High Sensitivity Kit (Active Motif), accordingly to the supplier's recommendations. Volumes and durations of reactions were adapted to the weak amount of tissue recovered at this stage (5-20 mg tissue per sample). Briefly, embryonic tissues were fixed in 1\% PFA then sonicated. Chromatin was incubated overnight with $2 \mu \mathrm{g}$ of rabbit anti-CDX2 (Abcam; ab76541; RRID:AB_1523334). The antibodybound protein/DNA complexes were precipitated using protein $\mathrm{G}$ agarose beads. After DNA purification on the DNA purification column, PCR reactions were performed on input and immunoprecipitated DNA for 38 cycles using primers flanking the Cdx2-binding site within the HoxA5 promoter: CGCTTTAACCCCTTTCAATTAC/ GCTAGGCCCAACCCTGTTAG ${ }^{15}$, or as negative control with primers corresponding to a genomic segment of the second intron of the $C d x 2$ gene, devoid of $C d x 2$ binding site: TGGGGCAATCTTAATGGGTA/ TGTAGCCTTGACTTGGCTTT $^{19}$. Quantification of the ChIP was performed by Sybr Green qPCR with the above HoxA5 primers and the FastStart Universal SYBR Green Master kit (Roche), using the $\Delta \Delta \mathrm{Ct}$ method relative to the Input and fixed at 1 for the value obtained in the samples of the trunk.

\section{Acknowledgements}

This work was supported by INSERM and the Ligue du Haut-Rhin contre le Cancer. We thank Prof S Britsch (UIm University, Germany) for kindly providing the anti-Gbx1 antibody and Dr G Page (Cell Marquen Sigma-Aldrich) for the anti-Sox10 antibody.

\section{Author details}

'Université de Strasbourg, Inserm, IRFAC/UMR-S1113, FMTS, 67200 Strasbourg, France. ${ }^{2}$ Karlsruhe Institute of Technology, Institute of Toxicology and Genetics, 76021 Karlsruhe, Germany. ${ }^{3}$ Institut Clinique de la Souris, 67404 IIIkirch Cedex, France

\section{Data availability}

RNAseq data are deposited in the GEO database under the accession number GSE123559.

\section{Conflict of interest}

The authors declare that they have no conflict of interest.
Publisher's note

Springer Nature remains neutral with regard to jurisdictional claims in published maps and institutional affiliations.

Supplementary Information accompanies this paper at (https://doi.org/ 10.1038/s41419-019-2040-0).

Received: 20 July 2019 Revised: 27 September 2019 Accepted: 3 October 2019

Published online: 24 October 2019

\section{References}

1. Dubourg, C. et al. Holoprosencephaly. Orphanet. J. Rare Dis. 2, 8 (2007).

2. Roessler, E. \& Muenke, M. The molecular genetics of holoprosencephaly. Am. J. Med. Genet C 154C, 52-61 (2010).

3. Minoux, M. \& Rijli, F. M. Molecular mechanisms of cranial neural crest cell migration and patterning in craniofacial development. Development 137 2605-2621 (2010).

4. Tam, P. P. L. et al. Formation of the embryonic head in the mouse: attributes of a gene regulatory network. Curr. Top. Dev. Biol. 117, 497-521 (2016).

5. Neijts, R., Simmini, S., Giuliani, F., van Rooijen, C., DeschampsJ. Region-specific regulation of posterior axial elongation during vertebrate embryogenesis. Dev. Dyn. 243, 88-98 (2014).

6. Mallo, M., Wellik, D. M. \& Deschamps, J. Hox genes and regional patterning of the vertebrate body plan. Dev. Biol. 344, 7-15 (2010).

7. Deschamps, J. \& Duboule, D. Embryonic timing, axial stem cells, chromatin dynamics, and the Hox clock. Genes Dev. 31, 1406-1416 (2017).

8. van Rooijen, C. et al. Evolutionarily conserved requirement of $\mathrm{Cdx}$ for postoccipital tissue emergence. Development 139, 2576-2583 (2012).

9. Aires, R. et al. Oct4 is a key regulator of vertebrate trunk length diversity. Dev. Cell. 38, 262-274 (2016).

10. Wellik, D. M. Hox genes and vertebrate axial pattern. Curr. Top. Dev. Biol. 88, 257-278 (2009).

11. Subramanian, V., Meyer, B. I. \& Gruss, P. Disruption of the murine homeobox gene $\mathrm{Cdx} 1$ affects axial skeletal identities by altering the mesodermal expression domains of Hox genes. Cell 83, 641-653 (1995).

12. Chawengsaksophak, K., James, R., Hammond, V. E., Kontgen, F. \& Beck, F. Homeosis and intestinal tumours in Cdx2 mutant mice. Nature 385, 84-87 (1997).

13. Prince, V. \& Lumsden, A. Hoxa-2 expression in normal and transposed rhombomeres: independent regulation in the neural tube and neural crest. Development 120, 911-923 (1994).

14. Amin, S. et al. Cdx and T brachyury co-activate growth signaling in the embryonic axial progenitor niche. Cell Rep. 17, 3165-3177 (2016).

15. Neijts, R., Amin, S., van Rooijen, C. \& Deschamps, J. Cdx is crucial for the timing mechanism driving colinear Hox activation and defines a trunk segment in the Hox cluster topology. Dev. Biol. 422, 146-154 (2017).

16. Strumpf, D. et al. Cdx2 is required for correct cell fate specification and differentiation of trophectoderm in the mouse blastocyst. Development 132, 2093-2112 (2005).

17. Huang, D. et al. The role of $\mathrm{Cd} \times 2$ as a lineage specific transcriptional repressor for pluripotent network during the first developmental cell lineage segregation. Sci. Rep. 7, 17156 (2017)

18. Gao, N., White, P. \& Kaestner, K. H. Establishment of intestinal identity and epithelial-mesenchymal signaling by Cdx2. Dev. Cell. 16, 588-599 (2009).

19. Stringer, E. J. Cdx2 determines the fate of postnatal intestinal endoderm. Development 139, 465-474 (2012).

20. Patau, K, Smith, D. W., Therman, E., Inhorn, S. L. \& Wagner, H. P. Multiple congenital anomaly caused by an extra autosome. Lancet 1, 790-793 (1960).

21. Choi, L. A. et al. Partial Trisomy 13 (Patau Syndrome) - An Autopdy Report. Korean J. Pathol. 36, 338-340 (2002).

22. Firth, H. V. et al. DECIPHER: database of chromosomal imbalance and phenotype in humans using ensembl resources. Am. J. Hum. Genet. 84, 524-533 (2009).

23. Peroos, S. et al. Longevity and Patau syndrome: what determines survival? BMJ Case Rep. https://doi.org/10.1136/bcr-06-2011-4381 (2012).

24. Liu, J.P. The function of growth/differentiation factor 11 (Gdf11) in rostrocaudal patterning of the developing spinal cord. Development 133 2865-2874 (2006) 
25. Tabaries, S. et al. Cdx protein interaction with Hoxa5 regulatory sequences contributes to Hoxa5 regional expression along the axial skeleton. Mol. Cell. Biol. 25, 1389-1401 (2005).

26. Wolf, L. et al. Histone posttranslational modifications and cell fate determination: lens induction requires the lysine acetyltransferases CBP and p300. Nucleic Acids Res. 41, 10199-10214 (2013).

27. Creuzet, S., Couly, G., Vincent, C. \& Le Douarin, N. M. Negative effect of Hox gene expression on the development of the neural crest-derived facial skeleton. Development 129, 4301-4313 (2002).

28. Kitazawa, T. et al. Distinct effects of Hoxa2 overexpression in cranial neural crest populations reveal that the mammalian hyomandibular-ceratohyal boundary maps within the styloid process. Dev. Biol. 402, 162-174 (2015).

29. Zaffran, S., Odelin, G., Stefanovic, S., Lescroart, F. \& Etchevers, H. C. Ectopic expression of Hoxb1 induces cardiac and craniofacial malformations. Genesis 56, e23221 (2018)

30. van den Akker, E. et al. Cdx1 and Cdx2 have overlapping functions in anteroposterior patterning and posterior axis elongation. Development $\mathbf{1 2 9}$ 2181-2193 (2002)

31. Mlodzik, M., Gibson, G. \& Gehring, W. J. Effects of ectopic expression of caudal during Drosophila development. Development 109, 271-277 (1990).

32. Silberg, D. G. et al. Cdx2 ectopic expression induces gastric intestinal metaplasia in transgenic mice. Gastroenterology 122, 689-696 (2002).

33. Banerjee, K. K. et al. Enhancer, transcriptional, and cell fate plasticity precedes intestinal determination during endoderm development. Genes Dev. 32 1430-1442 (2018).

34. Saxena, M. et al. Transcription factor-dependent 'anti-repressive' mammalian enhancers exclude H3K27me3 from extended genomic domains. Genes Dev. 31, 2391-2404 (2017).

35. Balbinot, C. et al. Fine-tuning and autoregulation of the intestinal determinant and tumor suppressor homeobox gene CDX2 by alternative splicing. Cell Death Differ. 24, 2173-2186 (2017).

36. Arnold, K. et al. Sox2(+) adult stem and progenitor cells are important for tissue regeneration and survival of mice. Cell Stem Cell 9, 317-329 (2011).
37. Madisen, L. et al. A robust and high-throughput Cre reporting and characterization system for the whole mouse brain. Nat. Neurosci. 13, 133-140 (2010).

38. Joyner, A. \& Wall, N. Immunohistochemistry of whole-mount mouse embryos. C. S. H. Protoc. 2008, pdb.prot4820 (2008).

39. Balbinot, $\mathrm{C}$. et al. The $\mathrm{Cdx} 2$ homeobox gene suppresses intestinal tumorigenesis through non-cell-autonomous mechanisms. J. Exp. Med. 215, 911-926 (2018).

40. John, A., Wildner, H. \& Britsch, S. The homeodomain transcription factor Gbx1 identifies a subpopulation of late-born GABAergic interneurons in the developing dorsal spinal cord. Dev. Dyn. 234, 767-771 (2005).

41. De Santa Barbara, P. et al. Direct interaction of SRY-related protein SOX9 and steroidogenic factor 1 regulates transcription of the human anti-Müllerian hormone gene. Mol. Cell. Biol. 18, 6653-6665 (1998).

42. Dobin, A. et al. STAR: ultrafast universal RNA-seq aligner. Bioinforma Oxf. Engl. 29, 15-21 (2013).

43. Anders, S., Pyl, P. T. \& Huber, W. HTSeq-a Python framework to work with high-throughput sequencing data. Bioinf. Oxf. Engl. 31, 166-169 (2015).

44. Love, M. I., Huber, W. \& Anders, S. Moderated estimation of fold change and dispersion for RNA-seq data with DESeq2. Genome Biol. 15, 550 (2014).

45. Huang, D. W., Sherman, B. T. \& Lempicki, R. A. Systematic and integrative analysis of large gene lists using DAVID bioinformatics resources. Nat. Protoc. $\mathbf{4}$, 44-57 (2009).

46. Heinz, S. et al. Simple combinations of lineage-determining transcription factors prime cis-regulatory elements required for macrophage and B cell identities. Mol. Cell 38, 576-589 (2010).

47. Khan, A. et al. JASPAR 2018: update of the open-access database of transcription factor binding profiles and its web framework. Nucleic Acids Res. 46, D260-D266 (2018).

48. Gupta, S., Stamatoyannopoulos, J. A., Bailey, T. L. \& Noble, W. S. Quantifying similarity between motifs. Genome Biol. 8, R24 (2007). 